\title{
Analysis of Bifurcation and Chaos of the Size-dependent Micro-plate Considering Damage
}

https://doi.org/10.1515/nleng-2018-0061

Received March 26, 2018; revised June 2, 2018; accepted August 3, 2018.

\begin{abstract}
In this research, nonlinear dynamics and characteristics of a micro-plate system under electrostatic forces on both sides are studied. A novel model, which takes micro-scale effect and damage effect into account, is established on the basis of the Talreja's tensor valued internal state damage model and modified couple stress theory. According to Hamilton principle, the dynamic governing equations of the size-dependent micro-plate are derived by variational method and solved via Galerkin method and the fourth order Runge-Kutta method. The effects of damage variable and material length scale parameter on bifurcation and chaos of the micro-plate system are presented with numerical simulations using the bifurcation diagram, Poincare map. Results provide a theoretical basis for the design of dynamic stability of electrically actuated micro- structures.
\end{abstract}

Keywords: MEMS; micro-plate; chaos; size effect; damage

\section{Introduction}

As Micro-electromechanical System (MEMS) received an extensive application, for example: micro-cantilever switch [1], nano/micro- resonator [2-6], micro-rotor [7], in areas such as biological technologies, communications, aerospace due to its superior properties: miniaturization, low energy consumption and increased reliability. Considerable amount of attention of researchers have been drawn in recent decades.

\footnotetext{
Xiumei Wang, School of Vehicle engineering (rail transit) college, Changzhou Vocational Institute of Mechatronic Technology, Changzhou 213164, Jiangsu, China

*Corresponding Author: Jihai Yuan, School of Civil Engineering and Architecture, Xiamen University of Technology, Xiamen 361024, Fujian, China, E-mail: traveller868789@126.com

Haorui Zhai, School of Automotive Engineering, Yancheng Institute of Technology, Yancheng 224051?Jiangsu, China
}

Micro-scale structure such as micro-plate has been widely used in MEMS such as micro-bumps, micro-mirrors and micro-phones [8]. However, the very existence of nonlinearity of these micro-devices due to nonlinear coupling between the forcing fields, squeezing film damping and mid-plane stretching have a significant influence on their performances. These nonlinearities often lead to nonlinear behaviors of MEMS device. Numerous investigations of nonlinear characteristics of micro-plate have been carried out in recent years. With consideration of electric force nonlinearity, Zhao [9] developed a new mode of electrically actuated micro-plate which is validated experimentally and further applied it into the investigation of pull-in instability. The static and dynamic behaviors of a rectangular micro-plate with nonlinearity of mid-plane stretching under electric force are explored by Saghir [10]. Based on von Karman assumption, Batra [11] studied pullin phenomenon of a clamped micro-plate subjected to van der Waals force. On the basis of the same assumption, Batra [12] also investigated pull-in instability of electrically actuated circular and rectangular micro-plates under electric force and Casimir force. The nonlinear dynamic properties of air-bearing slider were studied by Chen [13].Via Galerkin method and second order multiple scales method, Miandoab [14] investigated the nonlinear dynamic property of micro- resonators.

Although plentiful researches have been carried out by lots of knowledgeable scholars in this area, behavior of micro-structure is proven experimentally to be size dependent [15]. For this reason, classical continuum theory failed to capture micro-scale effect. With consideration of such inevitable factor in micro-structure, some investigations about bending response, nonlinear vibration and dynamic pull-in were reported in recent years. A size-dependent model was proposed by Tahani [16], who employed modified couple stress theory (MCST) [17] to study free vibration of pre-deformed micro-plates. As for the pull-in phenomenon peculiar to MEMS device, Askari [18] examined dynamic pull-in phenomenon of a size-dependent micro-plate on the basis of MCST. Wang [19] developed a reduced-order model incorporating nonlinearity, micro-scale effect and surface energy for free vibration and pull-in of micro/nano-plates subject to 
Casimir force. Nonlinear static and dynamic characteristics of electrically actuated size- dependent micro-plates were presented by Farokhi [20]. A size-dependent nonlinear mathematical model was proposed by Zheng [21], who employed MSCT to study nonlinear static behavior of piezoelectric cantilever micro- beams.

Meanwhile, defects such as damage and micro-cracks take place when MEMS devices work under complex environment. When subjected to undesirable external loads, these defects may evolve, as a result, leading to failure of MEMS devices. The very existences of such defects are one of the main reasons why fatigue damage [22], discrete brittle damage [23] and contact damage [24] take place during manufacturing and operating process. Therefore, it is significant to make a better understanding on the influence of these defects on the physical properties of these microstructures.

Though the mechanical properties of micro- plates have been studied by many researchers, the research efforts devoted to analysis of bifurcation and chaos of electrostatically actuated size-dependent micro-plate with damage are very rare. The aim of this work is to study bifurcation and chaos of micro-plate under electrostatic actuation. In this work, a novel model for size-dependent microplate with damage on the basis of the Talreja's tensor valued internal state damage model [25] and modified couple stress theory is developed. Hamilton's principle plays a decisive role in deriving the nonlinear dynamic equations. Galerkin method is used to transform the partial differential equations into ordinary differential equations in terms of time which are further solved employing the fourth order Runge-Kutta method. The impact of system parameters on bifurcation and chaos of the micro-plate is discussed in detail.

\section{Basic Equations}

\subsection{Constitutive relation with damage}

According to Talreja [25] and Chen [26], the Cauchy stresses can be given as:

$$
\sigma=\left\{\begin{array}{c}
\sigma_{x} \\
\sigma_{y} \\
\sigma_{x y}
\end{array}\right\}=Q^{d} \cdot\left\{\begin{array}{c}
\varepsilon_{x} \\
\varepsilon_{y} \\
\varepsilon_{x y}
\end{array}\right\}=\left[C_{0}+C_{d}\right] \cdot\left\{\begin{array}{c}
\varepsilon_{x} \\
\varepsilon_{y} \\
\varepsilon_{x y}
\end{array}\right\}
$$

where $C_{0}$ and $C_{d}$ are stiffness matrixes without damage and with damage correspondently and expressed as:

$$
\begin{gathered}
C_{0}=\left[\begin{array}{ccc}
Q_{11} & Q_{12} & 0 \\
& Q_{22} & 0 \\
& & Q_{66}
\end{array}\right]=\left[\begin{array}{ccc}
\frac{E}{1-v^{2}} & \frac{v E}{1-\nu} & 0 \\
& \frac{E}{1-v^{2}} & 0 \\
& & G
\end{array}\right] \\
C_{d}=\left[\begin{array}{ccc}
d_{11} & d_{12} & 0 \\
& d_{22} & 0 \\
& & d_{66}
\end{array}\right] D
\end{gathered}
$$

where $D$ is the damage variable which is a constant value and varies from 0 to 1 [28]; $d_{i j}$ are material parameters related to damage; $E, v$ and $G$ represent elastic modulus, Poisson ratio and shear modulus respectively.

\subsection{Basic Equations of micro-plate}

Using the coordinate system in Fig. 1, where a micro-plate has length $a$, width $b$, thickness $h . d$ and $V_{1}, V_{2}$ are the initial gap and the electric loads between the micro-plate and stationary electrodes. Assume that the shaded area in Fig. 2 is damaged. The position of damage is represented by the center $(e, f)$ of the damaged area and $S_{d}$ donates the ratio of damage area to total area of the micro-plate.

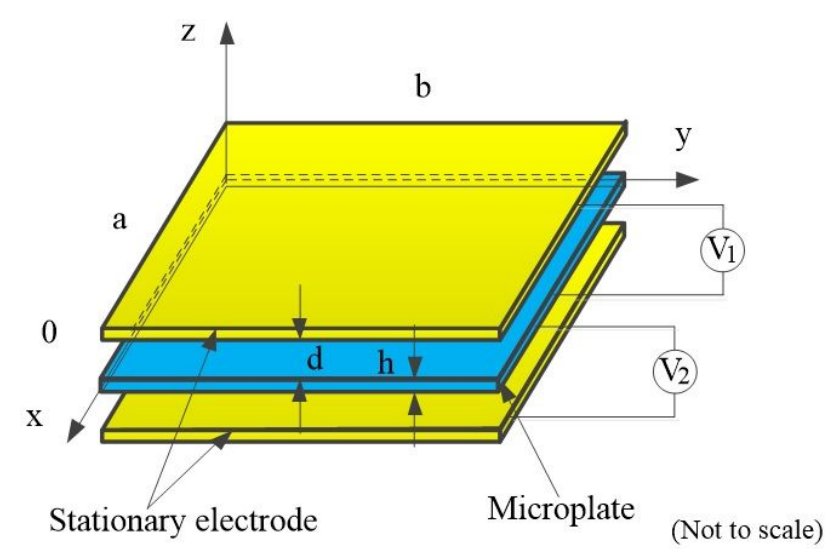

Fig. 1: Electrically actuated micro-plate.

According to the modified couple stress theory (Yang et al. 2002), the strain energy can be written as:

$$
U=\frac{1}{2} \int_{V}\left(\sigma_{i j} \varepsilon_{i j}+m_{i j} \chi_{i j}\right) d V \quad(i, j=x, y, z)
$$

where $V$ is the volume of the micro-plate; curvature tensor $\left(\chi_{i j}\right)$, stress tensor $\left(\varepsilon_{i j}\right)$, strain tensor $\left(\sigma_{i j}\right)$ and couple stress tensor $\left(m_{i j}\right)$ are defined as:

$$
\chi_{i j}=\frac{1}{2}\left(\theta_{i, j}+\theta_{j, i}\right)
$$




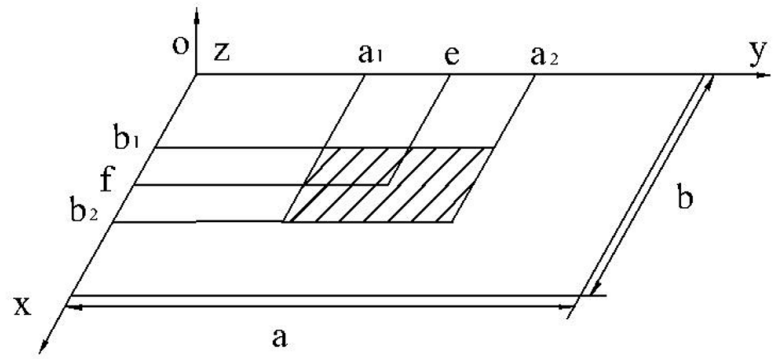

Fig. 2: Geometry configuration of the damaged micro-plate.

$$
\begin{gathered}
\varepsilon_{i j}=\frac{1}{2}\left(u_{i, j}+u_{j, i}\right) \\
\sigma_{i j}=\lambda \operatorname{tr}\left(\varepsilon_{i j}\right) I+2 \mu \varepsilon_{i j} \\
m_{i j}=2 l^{2} \mu \chi_{i j}
\end{gathered}
$$

where $\lambda$ and $\mu$ are lame constants; $l$ is material length scale parameter which is used for describing micro-scale effect; $u_{i}$ is displacement vector. The gyration vector $\left(\theta_{i}\right)$ is given as:

$$
\theta_{i}=\frac{1}{2} e_{i j k} u_{k, j}
$$

where $e_{i j k}$ is the replacement of tensor.

Based on von Karman plate theory, the displacements $u_{x}, u_{y}, u_{z}$ in $\backslash\left[\left\{u_{-} \mathbf{x}\right\},\left\{u_{-} \mathbf{y}\right\},\left\{u_{-} z\right\} \backslash\right]$ directions can be respectively given as

$$
\begin{aligned}
& u_{x}(x, y, z, t)=u(x, y, t)-z w_{, x} \\
& u_{y}(x, y, z, t)=v(x, y, t)-z w, y \\
& u_{z}(x, y, z, t)=w(x, y, t)
\end{aligned}
$$

where $u, v, w$ are the displacements of the neutral surface. The $\operatorname{strains}\left(\varepsilon_{x}^{0}, \varepsilon_{y}^{0}, \varepsilon_{x y}^{0}\right)$ of the neutral surface which are coupled with geometric nonlinearity and curvatures $\left(\kappa_{x}, \kappa_{y}, \kappa_{x y}\right)$ of the neutral surface are

$$
\begin{aligned}
& \varepsilon_{x}^{0}=u_{, x}+\frac{1}{2} w_{, x}^{2}, \\
& \varepsilon_{y}^{0}=v_{, y}+\frac{1}{2} w_{, y}^{2}, \\
& \varepsilon_{x y}^{0}=u_{, y}+v_{, x}+w_{, x} w_{, y}, \\
& \kappa_{x}=-w_{, x x}, \\
& \kappa_{y}=-w_{, y y}, \\
& \kappa_{x y}=-2 w_{, x y}
\end{aligned}
$$

The strains of the micro-plate are

$$
\begin{aligned}
& \varepsilon_{x}=\varepsilon_{x}^{0}+z \kappa_{x}, \\
& \varepsilon_{y}=\varepsilon_{y}^{0}+z \kappa_{y}, \\
& \varepsilon_{x y}=\varepsilon_{x y}^{0}+z \kappa_{x y}
\end{aligned}
$$

Substituting Eq. (10) into Eq. (9) yields gyration components

$$
\begin{aligned}
& \theta_{x}(x, y)=w_{, y}, \\
& \theta_{y}(x, y)=-w_{, x}, \\
& \theta_{z}(x, y)=\frac{1}{2}\left(v_{, x}-u, y\right)
\end{aligned}
$$

Substituting Eq. (13) into Eq. (5), we obtain curvature components

$$
\begin{aligned}
& \chi_{x x}=w_{, x y}, \\
& \chi_{y y}=-w_{, x y}, \\
& \chi_{x y}=\frac{1}{2}\left(w_{, y y}-w_{, x x}\right), \\
& \chi_{x z}=\frac{1}{4}\left(v_{, x x}-u, x y\right), \\
& \chi_{y z}=\frac{1}{4}\left(v_{, x y}-u_{, y y}\right)
\end{aligned}
$$

When the lame constant are replaced by Young's modulus and Poisson's ratio, the physical relations can be given as follow:

$$
\left\{\begin{array}{l}
\sigma_{x} \\
\sigma_{y} \\
\sigma_{x y}
\end{array}\right\}=Q^{d}\left\{\begin{array}{l}
\varepsilon_{x} \\
\varepsilon_{y} \\
\varepsilon_{x y}
\end{array}\right\}
$$

$\left(m_{x x}, m_{y y}, m_{x y}, m_{x z}, m_{y z}\right)=F\left(\chi_{x x}, \chi_{y y}, \chi_{x y}, \chi_{x z}, \chi_{y z}\right)$

where

$$
\begin{aligned}
& Q^{d}=\left[\begin{array}{ccc}
\frac{E}{1-v^{2}} & \frac{v E}{1-v^{2}} & 0 \\
& \frac{E}{1-v^{2}} & 0 \\
& G
\end{array}\right]+\left[\begin{array}{ccc}
d_{11} & d_{12} & 0 \\
& d_{22} & 0 \\
& & d_{66}
\end{array}\right] D \\
& F=2 l^{2}\left(G+d_{66} D\right)
\end{aligned}
$$

Note that the damage variable is constant through the thickness of plate [27]. The membrane stress resultants $N_{x}, N_{y}, N_{x y}$, bending moments $M_{x}, M_{y}, M_{x y}$ and couple moments $Y_{x x}, Y_{y y}, Y_{x y}, Y_{x z}, Y_{y z}$ are

$$
\begin{aligned}
& {\left[\begin{array}{lllll}
N_{x} & N_{y} & N_{x y}
\end{array}\right]=\int_{-\frac{h}{2}}^{\frac{h}{2}}\left[\sigma_{x} \sigma_{y} \sigma_{x y}\right] d z} \\
& {\left[\begin{array}{llllll}
M_{x} & M_{y} & M_{x y}
\end{array}\right]=\int_{-\frac{h}{2}}^{\frac{h}{2}} z\left[\begin{array}{llll}
\sigma_{x} & \sigma_{y} & \sigma_{x y}
\end{array}\right] d z} \\
& {\left[\begin{array}{llllll}
Y_{X X} & Y_{y y} & Y_{x y} & Y_{x z} & Y_{y z}
\end{array}\right]=} \\
& \int_{-\frac{h}{2}}^{\frac{h}{2}}\left[\begin{array}{llllll}
m_{x x} & m_{y y} & m_{x y} & m_{x z} & m_{y z}
\end{array}\right] d z
\end{aligned}
$$

Substituting Eq. (15) and Eq. (16) into Eq. (18), we obtain

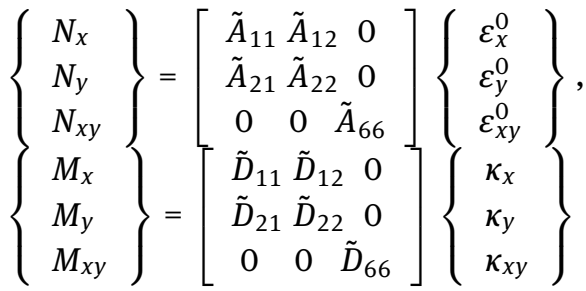

$$
\begin{aligned}
& {\left[\begin{array}{lllll}
Y_{x x} & Y_{y y} & Y_{x y} & Y_{x z} & Y_{y z}
\end{array}\right]=} \\
& \tilde{G} \quad\left[\begin{array}{lllll}
\chi_{x x} & \chi_{y y} & \chi_{x y} & \chi_{x z} & \chi_{y z}
\end{array}\right]
\end{aligned}
$$


The membrane stiffness coefficients $\tilde{A}_{i j}(i, j=1,2,6)$, the bending stiffness coefficients $\tilde{D}_{i j}$ and the contribution of rotation gradients to the bending stiffness coefficient $\tilde{G}$, which is also called as additional bending stiffness coefficient, of the micro-plate can be defined as:

$$
\begin{aligned}
& \tilde{A}_{i j}=\int_{-\frac{h}{2}}^{\frac{h}{2}}\left(C_{i j}^{0}+C_{i j}^{d}\right) d z=A_{i j}+\Delta A_{i j} \\
& \tilde{D}_{i j}=\int_{-\frac{h}{2}}^{\frac{h}{2}} z^{2}\left(C_{i j}^{0}+C_{i j}^{d}\right) d z=D_{i j}+\Delta D_{i j} \\
& \tilde{G}=\int_{-\frac{h}{2}}^{\frac{h}{2}} F d z=G+\Delta G
\end{aligned}
$$

where $A_{i j}, D_{i j}$ and $G$ respectively donate the membrane stiffness coefficients, the bending stiffness coefficients and the additional bending stiffness coefficient of a perfect micro-plate; $\Delta A_{i j}, \Delta D_{i j}$ and $\Delta G$ represent the decrease of the membrane stiffness coefficients, the bending stiffness coefficients and the additional bending stiffness coefficient on account of damage respectively.

It is known that principle of Hamilton can be written as:

$$
\delta \int_{t_{1}}^{t_{2}}(T-U+W) d t=0
$$

Substituting Eqs. (12), (14) - (16) into Eq. (4), the virtual strain energy of the micro-plate is given as

$$
\begin{aligned}
\delta U & =\int_{V}\left(\sigma_{i j} \delta \varepsilon_{i j}\right) d V+\int_{V}\left(m_{i j} \delta \chi_{i j}\right) d V \\
& =\int_{V}\left(\sigma_{x x} \delta \varepsilon_{x x}+\sigma_{y y} \delta \varepsilon_{y y}+\sigma_{x y} \delta \varepsilon_{x y}\right) d V \\
& +\int_{V}\left(m_{x x} \delta \chi_{x x}+m_{y y} \delta \chi_{y y}+2 m_{x y} \delta \chi_{x y}\right. \\
& \left.+2 m_{x z} \delta \chi_{x z}+2 m_{y z} \delta \chi_{y z}\right) d V
\end{aligned}
$$

The virtual kinetic energy of the micro-plate is

$$
\delta T=\frac{1}{2} \rho \int_{V} \delta\left(w_{, t}\right)^{2} d V
$$

where $\rho$ is mass density of micro-plate.

And the virtual work performed by external forces can be written as

$$
W=\iint_{S}\left(F_{z}-c w, t\right) \partial w d S
$$

where $c$ is the damping coefficient of air in the gap; $F_{z}$ is electric force which is considered as transverse load and can be defined as

$$
F_{z}=\frac{\varepsilon_{v}}{2}\left[\frac{V_{1}^{2}}{(d-w)^{2}}+\frac{V_{2}^{2}}{(d+w)^{2}}\right]
$$

where $\varepsilon_{v}$ is air permittivity constant between the microplate and stationary electrodes. Here, the voltages are composed of two parts:

$$
V_{1}=V_{D C}+V_{A C} \cos \Omega t \quad V_{2}=V_{D C}-V_{A C} \cos \Omega t
$$

where $V_{D C}$ and $V_{A C}$ are DC voltage and amplitude of AC voltage, respectively; $\Omega$ donates the frequency of the AC voltage.

Substituting Eqs. (22) - (24) into Eq. (21), the nonlinear governing differential equations of the size-dependent micro-plate can be obtained as

$$
\begin{aligned}
& N_{x, x}+N_{x y, y}+\frac{1}{2} Y_{x z, x y}+\frac{1}{2} Y_{y z, y y}=0 \\
& N_{y, y}+N_{x y, x}-\frac{1}{2} Y_{x z, x x}-\frac{1}{2} Y_{y z, x y}=0 \\
& -\left(N_{x} w_{, x}+N_{x y} w_{, y}\right)_{, x}-\left(N_{y} w_{, y}+N_{x y} w_{, x}\right)_{, y} \\
& +\left(-M_{x}-Y_{x y}\right)_{, x x}+\left(-M_{y}+Y_{x y}\right)_{, y y^{+}} \\
& \left(-2 M_{x y}+Y_{x x}-Y_{y y}\right)_{, x y}+F_{z}=\rho h w_{, t t}+c w_{, t}
\end{aligned}
$$

Boundary conditions:

$$
\begin{aligned}
& x=0, a \\
& N_{x}+\frac{1}{2} Y_{x z, y}=0 \text { or } \delta u=0, \\
& N_{x y}-\frac{1}{2} Y_{x z, x}=0 \text { or } \delta v=0, \\
& \frac{1}{2} Y_{x z}=0 \text { or } \delta v_{, x}=0, \\
& \frac{1}{2} Y_{y z}=0 \text { or } \delta v, y=0 \\
& N_{x} w_{, x}+N_{x y} w, y-\left(-M_{x}-Y_{x y}\right)_{, x} \\
& -\left(-2 M_{x y}+Y_{x x}-Y_{y y}\right)_{, y}=0 \text { or } \delta w=0 \\
& -M_{x}-Y_{x y}=0 \text { or } \delta w_{, x}=0 \\
& y=0, b \\
& N_{y}-\frac{1}{2} Y_{y z, x}=0 \text { or } \delta v=0, \\
& N_{x y}+\frac{1}{2} Y_{y z, y}=0 \text { or } \delta u=0, \\
& -\frac{1}{2} Y_{x z}=0 \text { or } \delta u, x=0, \\
& -\frac{1}{2} Y_{y z}=0 \text { or } \delta u, y=0, \\
& N_{y} w_{, y}+N_{x y} w_{, x}-\left(-M_{y}+Y_{x y}\right)_{, y} \\
& -\left(-2 M_{x y}+Y_{x x}-Y_{y y}\right)_{, x}=0 \text { or } \delta w=0 \\
& -M_{y}+Y_{x y}=0 \text { or } \delta w_{, y}=0
\end{aligned}
$$

Introducing the following dimensionless parameters:

$$
\begin{aligned}
& \xi=\frac{x}{a}, \eta=\frac{y}{b}, U=\frac{a u}{h^{2}}, V=\frac{a v}{h^{2}}, W=\frac{w}{h}, \lambda=\frac{h}{d}, \\
& \lambda_{1}=\frac{a}{b}, \lambda_{2}=\frac{a}{h}, \bar{A}_{i j}=\frac{A_{i j}}{Q_{11} h}, \bar{l}=\frac{l}{h}, \Delta \bar{A}_{i j}=\frac{\Delta A_{i j}}{Q_{11} h}, \\
& \bar{D}_{i j}=\frac{D_{i j}}{Q_{11} h^{3}}, \Delta \bar{D}_{i j}=\frac{\Delta D_{i j}}{Q_{11} h^{3}}, \bar{G}_{1}=\frac{G}{8 G_{12} a^{3}}, \\
& \bar{G}_{2}=\frac{G}{8 G_{12} a^{3}}, \bar{G}_{3}=\frac{G}{2 G_{12} h^{3}}, \Delta \bar{G}_{1}=\frac{\Delta G}{8 G_{12} a^{3}}, \\
& \Delta \bar{G}_{2}=\frac{\Delta G}{8 G_{12} a^{3}}, \Delta \bar{G}_{3}=\frac{\Delta G}{2 G_{12} h^{3}}, \bar{d}_{i j}=\frac{d_{i j}}{Q_{11}}, \\
& \beta=\frac{G_{12}}{Q_{11}}, Q_{11}=\frac{E}{1-v^{2}}, \tau=\frac{t}{T}, \alpha_{1}=\frac{\varepsilon_{v} a^{4} V_{0}^{2}}{d^{2} h^{4} Q_{11}}, \\
& \alpha_{2}=\frac{\varepsilon_{v} a^{4} V_{0}^{2}}{d^{3} h^{3} Q_{11}}, \bar{c}=\frac{c a^{4}}{Q_{11} h^{3} T}, p=\frac{\rho a^{4}}{Q_{11} h^{2} T^{2}}, \\
& \bar{\Omega}=\Omega T, \bar{V}_{C}=\frac{V_{c}}{V_{0}}
\end{aligned}
$$

in which $V_{0}$ is the unit voltage value.

After applying the Taylor series expansion to Eq. (25) and neglecting the nonlinear terms of series [1], the dimensionless nonlinear governing equations of the size- 
dependent micro-plate are obtained as:

$$
\begin{aligned}
& \left(\bar{A}_{11}+\Delta \bar{A}_{11}\right)\left(U_{, \xi \xi}+W_{, \xi} W_{, \xi \xi}\right) \\
& +\left(\bar{A}_{12}+\Delta \bar{A}_{12}\right)\left(\lambda_{1} V_{, \xi \eta}+\lambda_{1}^{2} W_{, \eta} W_{, \xi \eta}\right) \\
& +\left(\bar{A}_{66}+\Delta \bar{A}_{66}\right)\left(\lambda_{1}^{2} U_{, \eta \eta}+\lambda_{1} V_{, \xi \eta}+\lambda_{1}^{2} W_{, \xi} W_{, \eta \eta}+\lambda_{1}^{2} W_{, \eta} W_{, \xi \eta}\right) \\
& +\beta\left(\bar{G}_{1}+\Delta \bar{G}_{1}\right)\left(\lambda_{1} \lambda_{2} V_{, \xi \xi \xi \eta}-\lambda_{1}^{2} \lambda_{2} U_{, \xi \xi \eta \eta}+\lambda_{1}^{3} \lambda_{2} V_{, \xi \eta \eta \eta}\right. \\
& \left.-\lambda_{1}^{4} \lambda_{2} U, \eta \eta \eta \eta\right)=0 \\
& \left(\bar{A}_{12}+\Delta \bar{A}_{12}\right)\left(\lambda_{1} U_{, \xi \eta}+\lambda_{1} W_{, \xi} W_{, \xi \eta}\right) \\
& +\left(\bar{A}_{22}+\Delta \bar{A}_{22}\right)\left(\lambda_{1}^{2} V_{, \eta \eta}+\lambda_{1}^{3} W_{, \eta} W_{, \eta \eta}\right) \\
& +\left(\bar{A}_{66}+\Delta \bar{A}_{66}\right)\left(\lambda_{1} U_{, \xi \eta}+V_{, \xi \xi}+\lambda_{1} W_{, \eta} W_{, \xi \xi}+\lambda_{1} W_{, \xi} W_{, \xi \eta}\right) \\
& -\beta\left(\bar{G}_{2}+\Delta \bar{G}_{2}\right)\left(\lambda_{2} V_{, \xi \xi \xi \xi}-\lambda_{1} \lambda_{2} U_{, \xi \xi \xi \eta}+\lambda_{1}^{2} \lambda_{2} V_{, \xi \xi \eta \eta}\right. \\
& \left.-\lambda_{1}^{3} \lambda_{2} U_{, \xi \eta \eta \eta \eta}\right)=0 \\
& {\left[\bar{D}_{11}+\Delta \bar{D}_{11}+\beta\left(\bar{G}_{3}+\Delta \bar{G}_{3}\right)\right] W_{, \xi \xi \xi \xi}+\left[2\left(\bar{D}_{12}+\Delta \bar{D}_{12}\right)\right.} \\
& \left.+4\left(\bar{D}_{66}+\Delta \bar{D}_{66}\right)+2 \beta\left(\bar{G}_{3}+\Delta \bar{G}_{3}\right)\right] \lambda_{1}^{2} W_{, \xi \xi \eta \eta}+p W_{, \tau \tau}+\bar{c} W_{, \tau} \\
& +\left[\bar{D}_{22}+\Delta \bar{D}_{22}+\beta\left(\bar{G}_{3}+\Delta \bar{G}_{3}\right)\right] \lambda_{1}^{4} W_{, \eta \eta \eta \eta} \\
& =\left[\left(\bar{A}_{11}+\Delta \bar{A}_{11}\right)\left(U_{, \xi}+\frac{1}{2} W_{, \xi}^{2}\right)+\left(\bar{A}_{12}+\Delta \bar{A}_{12}\right)\left(\lambda_{1} V_{, \eta}\right.\right. \\
& \left.\left.+\frac{1}{2} \lambda_{1}^{2} W_{, \eta}^{2}\right)\right] W_{, \xi \xi}+\left[\left(\bar{A}_{11}+\Delta \bar{A}_{11}\right)\left(U_{, \xi \xi}+W_{, \xi} W_{, \xi \xi}\right)\right. \\
& \left.+\left(\bar{A}_{12}+\Delta \bar{A}_{12}\right)\left(\lambda_{1} V_{, \xi \eta}+\lambda_{1}^{2} W_{, \eta} W_{, \xi \eta}\right)\right] W_{, \xi} \\
& +2\left(\bar{A}_{66}+\Delta \bar{A}_{66}\right)\left(\lambda_{1}^{2} U_{, \eta}+\lambda_{1} V_{, \xi}+\lambda_{1}^{2} W_{, \eta} W_{, \xi}\right) W_{, \xi \eta} \\
& +\left(\bar{A}_{66}+\Delta \bar{A}_{66}\right)\left(\lambda_{1} V_{, \xi \xi}+\lambda_{1}^{2} U_{, \xi \eta}+\lambda_{1}^{2} W_{, \eta} W_{, \xi \xi}\right. \\
& \left.+\lambda_{1}^{2} W_{, \xi} W_{, \xi \eta}\right) W_{, \eta \eta}+\left[\left(\bar{A}_{12}+\Delta \bar{A}_{12}\right)\left(\lambda_{1}^{2} U_{, \xi}+\frac{1}{2} \lambda_{1}^{2} W_{, \xi}^{2}\right)\right. \\
& \left.+\left(\bar{A}_{22}+\Delta \bar{A}_{22}\right)\left(\lambda_{1}^{3} V_{, \eta \eta}+\frac{1}{2} \lambda_{1}^{4} W_{, \eta}^{2}\right)\right] W_{, \eta \eta} \\
& +\left[\left(\bar{A}_{12}+\Delta \bar{A}_{12}\right)\left(\lambda_{1}^{2} U_{, \xi \eta}+\lambda_{1}^{2} W_{, \xi} W_{, \xi \eta}\right)\right. \\
& \left.+\left(\bar{A}_{22}+\Delta \bar{A}_{22}\right)\left(\lambda_{1}^{3} V_{, \eta \eta}+\lambda_{1}^{4} W_{, \eta} W_{, \eta \eta}\right)\right] W_{, \eta} \\
& +\left(\bar{A}_{66}+\Delta \bar{A}_{66}\right)\left(\lambda_{1}^{2} U_{, \eta \eta}+\lambda_{1} V_{, \xi \eta}+\lambda_{1}^{2} W_{, \xi \eta} W_{, \eta}\right. \\
& \left.+\lambda_{1}^{2} W_{, \xi} W_{, \eta \eta}\right) W_{, \xi}+\alpha_{1} \bar{V}_{D C} \bar{V}_{A C} \cos \Omega \bar{\Omega}_{2} \tau+\alpha_{2} \bar{V}_{D C}^{2} W
\end{aligned}
$$

With four edges are clamped, the dimensionless boundary conditions of the micro-plate are

$$
\begin{aligned}
& \xi=0,1 \quad V=0, \quad W=0, \quad V_{, \xi}=0, \quad V_{, \eta}=0, \\
& W_{, \xi}=0, \quad \bar{A}_{11}\left(U_{, \xi}+\frac{1}{2} W_{, \xi}^{2}\right)+\frac{\beta \bar{G}_{3}}{8}\left(\frac{h^{2}}{a b} V_{, \xi \xi \eta \eta}-\frac{h^{2}}{b^{2}} U_{, \xi \eta \eta \eta}\right)=0 \\
& \eta=0,1 \quad U=0, \quad W=0, U_{, \xi}=0, \quad U_{, \eta}=0, \quad W_{, \eta}=0, \\
& \bar{A}_{22}\left(V_{, \eta}+\frac{1}{2} \lambda_{1} W_{, \eta}^{2}\right)-\frac{\beta \bar{G}_{3}}{8}\left(\frac{h^{2}}{a^{2}} V_{, \xi \xi \eta}-\frac{h^{2}}{a b} U_{, \xi \eta \eta}\right)=0
\end{aligned}
$$

\section{Solution Methodologies}

Firstly, note that the items concluding material length scale parameter in the boundary conditions are so small compare with other items that they can be ignored in the calculation. A solution which satisfies the boundary conditions can be given as

$$
\begin{aligned}
& U(\xi, \eta, \tau)=\bar{U}(\tau) \cos \pi \xi \sin ^{2} \pi \eta \\
& V(\xi, \eta, \tau)=\bar{V}(\tau) \sin ^{2} \pi \xi \cos \pi \eta \\
& W(\xi, \eta, \tau)=\bar{W}(\tau) \sin ^{2} \pi \xi \sin ^{2} \pi \eta
\end{aligned}
$$

Submitting Eq. (33) into Eq. (31) and multi- plying them by $\cos \pi \xi \sin ^{2} \pi \eta, \sin ^{2} \pi \xi \cos \pi \eta$ and $\sin ^{2} \pi \xi \sin ^{2} \pi \eta$ respectively, and integ- rating them from 0 to 1 with respect to $\xi$ and $\eta$, then the nonlinear differential equations can be obtained as

$$
\begin{aligned}
& {\left[-\frac{3 \pi^{2}}{16} \bar{A}_{11}-\frac{\pi^{2} \lambda_{1}^{2}}{4} \bar{A}_{66}-\bar{G}_{1} \beta\left(\frac{\pi^{4}}{4} \lambda_{1}^{2} \lambda_{2}\right.\right.} \\
& \left.\left.+\pi^{4} \lambda_{1}^{4} \lambda_{2}\right)+\Delta L_{11}\right] \bar{U}+\left[-\frac{16}{9} \lambda_{1} \bar{A}_{12}-\frac{16}{9} \bar{A}_{66} \lambda_{1}\right. \\
& \left.+\bar{G}_{1} \beta\left(\frac{64 \pi^{2}}{9} \lambda_{1} \lambda_{2}+\frac{16 \pi^{2}}{9} \lambda_{1}^{3} \lambda_{2}\right)+\Delta L_{12}\right] \bar{V}+ \\
& {\left[\frac{\pi^{2}}{6} \bar{A}_{11}+\frac{2 \pi^{2}}{15} \lambda_{1}^{2} \bar{A}_{12}-\frac{2 \pi^{2}}{15} \bar{A}_{66} \lambda_{1}^{2}+\Delta L_{13}\right] \bar{W}^{2}=0} \\
& {\left[-\frac{16}{9} \lambda_{1} \bar{A}_{12}-\frac{16}{9} \lambda_{1} \bar{A}_{66}+\bar{G}_{2} \beta\left(\lambda_{1} \lambda_{2} \frac{16 \pi^{2}}{9}\right.\right.} \\
& \left.\left.+\lambda_{1}^{3} \lambda_{2} \frac{64 \pi^{2}}{9}\right)+\Delta L_{21}\right] \bar{U}+\left[-\frac{3 \pi^{2}}{16} \lambda_{1}^{2} \bar{A}_{22}\right. \\
& \left.-\frac{\pi^{2}}{4} \bar{A}_{66}-\bar{G}_{2} \beta\left(\frac{\pi^{4}}{4} \lambda_{1}^{2} \lambda_{2}+\pi^{4} \lambda_{2}\right)+\Delta L_{22}\right] \bar{V}+ \\
& {\left[\frac{2 \pi^{2}}{15} \lambda_{1} \bar{A}_{12}+\frac{\pi^{2}}{6} \lambda_{1}^{3} \bar{A}_{22}-\frac{2 \pi^{2}}{15} \bar{A}_{66} \lambda_{1}+\Delta L_{23}\right] \bar{W}^{2}=0}
\end{aligned}
$$

where $\Delta L_{i j}(i=1 \sim 3, j=1 \sim 4)$ are shown in Appendix.

For convenience, Eq. (34) can be rewritten as follows

$$
\begin{aligned}
& M_{11} \bar{U}+M_{12} \bar{V}+M_{13} \bar{W}^{2}=0 \\
& M_{21} \bar{U}+M_{22} \bar{V}+M_{23} \bar{W}^{2}=0
\end{aligned}
$$

$M_{31} \bar{W}+\frac{p}{4} \bar{W}_{, \tau \tau}+\frac{\bar{c}}{4} \bar{W}_{, \tau}=M_{32} \bar{U} \bar{W}+M_{33} \bar{V} \bar{W}$ $+M_{34} \bar{W}^{3}+\frac{1}{4} \alpha_{1} \bar{V}_{D C} \bar{V}_{A C} \cos \bar{\Omega} \tau+\frac{9}{64} \alpha_{2} \bar{V}_{D C}^{2} \bar{W}$

where

$$
\begin{aligned}
& M_{11}=-\frac{3 \pi^{2}}{16} \bar{A}_{11}-\frac{\pi^{2} \lambda_{1}^{2}}{4} \bar{A}_{66}-\bar{G}_{1} \beta\left(\frac{\pi^{4}}{4} \lambda_{1}^{2} \lambda_{2}\right. \\
& \left.+\pi^{4} \lambda_{1}^{4} \lambda_{2}\right)+\Delta L_{11} \\
& M_{12}=-\frac{16}{9} \lambda_{1} \bar{A}_{12}-\frac{16}{9} \bar{A}_{66} \lambda_{1}+\bar{G}_{1} \beta\left(\frac{64 \pi^{2}}{9} \lambda_{1} \lambda_{2}\right. \\
& \left.+\frac{16 \pi^{2}}{9} \lambda_{1}^{3} \lambda_{2}\right)+\Delta L_{12} \\
& M_{13}=\frac{\pi^{2}}{6} \bar{A}_{11}+\frac{2 \pi^{2}}{15} \lambda_{1}^{2} \bar{A}_{12}-\frac{2 \pi^{2}}{15} \bar{A}_{66} \lambda_{1}^{2}+\Delta L_{13} \\
& M_{21}=-\frac{16}{9} \lambda_{1} \bar{A}_{12}-\frac{16}{9} \lambda_{1} \bar{A}_{66}+\bar{G}_{2} \beta\left(\lambda_{1} \lambda_{2} \frac{16 \pi^{2}}{9}\right. \\
& \left.+\lambda_{1}^{3} \lambda_{2} \frac{64 \pi^{2}}{9}\right)+\Delta L_{21} \\
& M_{22}=-\frac{3 \pi^{2}}{16} \lambda_{1}^{2} \bar{A}_{22}-\frac{\pi^{2}}{4} \bar{A}_{66}-\bar{G}_{2} \beta\left(\frac{\pi^{4}}{4} \lambda_{1}^{2} \lambda_{2}\right. \\
& \left.+\pi^{4} \lambda_{2}\right)+\Delta L_{22} \\
& M_{23}=\frac{2 \pi^{2}}{15} \lambda_{1} \bar{A}_{12}+\frac{\pi^{2}}{6} \lambda_{1}^{3} \bar{A}_{22}-\frac{2 \pi^{2}}{15} \bar{A}_{66} \lambda_{1}+\Delta L_{23} \\
& M_{31}=\left[3\left(\bar{D}_{11}+\beta \bar{G}_{3}\right)+\lambda_{1}^{2}\left(2 \bar{D}_{12}+4 \bar{D}_{66}+2 \beta \bar{G}_{3}\right)\right. \\
& \left.+3 \lambda_{1}^{4}\left(\bar{D}_{22}+\beta \bar{G}_{3}\right)\right] \frac{\pi^{4}}{4}+\Delta L_{31} \\
& M_{32}=\frac{\pi^{2}}{3} \bar{A}_{11}-\frac{4 \pi^{2}}{15} \lambda_{1}^{2} \bar{A}_{66}+\frac{4 \pi^{2}}{15} \lambda_{1}^{2} \bar{A}_{12}+\Delta L_{32} \\
& M_{33}=\frac{4 \pi^{2}}{15} \lambda_{1} \bar{A}_{12}-\frac{4 \pi^{2}}{15} \lambda_{1} \bar{A}_{66}+\lambda_{1}^{3} \bar{A}_{22} \frac{\pi^{2}}{3}+\Delta L_{33} \\
& M_{34}=-\frac{52.5 \pi^{4}}{1024} \bar{A}_{11}-\frac{25 \pi^{4}}{1024} \lambda_{1}^{2} \bar{A}_{12}-\frac{52.5 \pi^{4}}{1024} \lambda_{1}^{4} \bar{A}_{22} \\
& -\frac{50 \pi^{4}}{1024} \bar{A}_{66} \lambda_{1}^{2}+\Delta L_{34}
\end{aligned}
$$

The unknown functions $\bar{U}(\tau), \bar{V}(\tau)$ can be expressed by $\bar{W}(\tau)$ after solving Eq. (35) and (36), then substituting 
the resultant expressions into Eq. (37), the nonlinear differential equation which is only related to $\bar{W}(\tau)$ can be obtained

$$
\begin{aligned}
& \frac{1}{4} p \bar{W}_{, \tau \tau}+\frac{1}{4} \bar{c} \bar{W}_{, \tau}+M_{31} \bar{W}-\varphi \bar{W}^{3}= \\
& \frac{1}{4} \alpha_{1} \bar{V}_{D C} \bar{V}_{A C} \cos \bar{\Omega} \tau+\frac{9}{64} \alpha_{2} \bar{V}_{D C}^{2} \bar{W}
\end{aligned}
$$

where

$$
\begin{aligned}
\varphi= & M_{34}+\frac{M_{32} M_{22} M_{13}-M_{32} M_{12} M_{23}}{M_{12} M_{21}-M_{11} M_{22}} \\
& +\frac{M_{33} M_{11} M_{23}-M_{33} M_{13} M_{21}}{M_{12} M_{21}-M_{11} M_{22}}
\end{aligned}
$$

Suppose that $x_{1}(\tau)=\bar{W}, x_{2}(\tau)=\dot{\dagger} W$, Eq. (38)can be written as

$$
\begin{aligned}
\dot{x}_{1}(\tau)= & x_{2}(\tau) \\
\dot{x}_{2}(\tau)=- & -\frac{\bar{c}}{p} x_{2}(\tau)-\frac{4}{p} M_{31} x_{1}(\tau)+\frac{4}{p} \varphi x_{1}^{3}(\tau) \\
& +\frac{4}{p}\left[\frac{1}{4} \alpha_{1} \bar{V}_{D C} \bar{V}_{A C} \cos \bar{\Omega} \tau+\frac{9}{64} \alpha_{2} \bar{V}_{D C}^{2} x_{1}(\tau)\right]
\end{aligned}
$$

\section{Solution and discussion}

In this section, dynamic characteristics of nonlinear motion of the micro-plate are investigated under the influence of size effect and damage. In the following numerical examples, the geometric parameters are taken as $a=$ $300 \mu \mathrm{m}, b=300 \mu \mathrm{m}, d=10 \mu \mathrm{m}, h=10 \mu \mathrm{m}$ and the physical parameters are set to $E=61 \mathrm{Gpa}, \varepsilon_{v}=8.854 \times$ $10^{-12} \mathrm{C}^{2} \mathrm{~N}^{-1} \mathrm{~m}^{-2}, v=0.35, \beta=0.3, p=1$ Here, the AC voltage frequency $\bar{\Omega}$, the AC voltage $\bar{V}_{A C}$ and the DC voltage $\bar{V}_{D C}$ are taken as $\pi / 4,20$ and 300 respectively. When the damage effect is taken into consideration, the material parameters related to damage in the numeral examples are [28]

$$
\begin{aligned}
& \bar{d}_{11}=-0.5, \quad \bar{d}_{12}=-0.25, \quad \bar{d}_{22}=-0.5, \\
& \bar{d}_{66}=-0.25, \quad S_{d}=16 \%
\end{aligned}
$$

When the non-autonomous system (Eq. (39)) is solved by the fourth order Runge-Kutta method, the corresponding initial conditions are set to $\left(x_{1}(\tau), x_{2}(\tau)\right)=(0,0)$. In order to avoid the numerical divergence and ensure a stable solution, the integration step is chosen to be 0.005 . Here, effect of two main parameters: damage variable and material length scale parameter on the dynamic behavior of the system are studied by using bifurcation diagram and Poincare maps in the numerical examples.

\subsection{Influence of damage}

In this section, we focus on the influence of damage on the dynamic behavior of the micro-plate system. Fig. 3 presents the bifurcation diagram of the micro-plate system using damage variable as the control parameter with nondimensional material length scale parameter $\bar{l}=0.1$ and damping coefficient $\bar{c}=0.003$.

Fig. 3 illustrates that the response of the micro- plate system transforms from periodicity to chaotic motion. It can be seen from Fig. 3 that: 1)the middle point amplitude of vibration of the micro-plate is becoming larger and larger with the increase of damage variable; 2)the microplate system is becoming more and more unstable as the damage variable increases. As shown in Fig. 4, when the damage variable is set to 0.003 , the Poincare map shows a single point which indicates periodic motion of the system; when the damage variable is set to 0.13 , the Poincare map shows fractal or chaotic characteristic which indicates chaotic motion of the system.

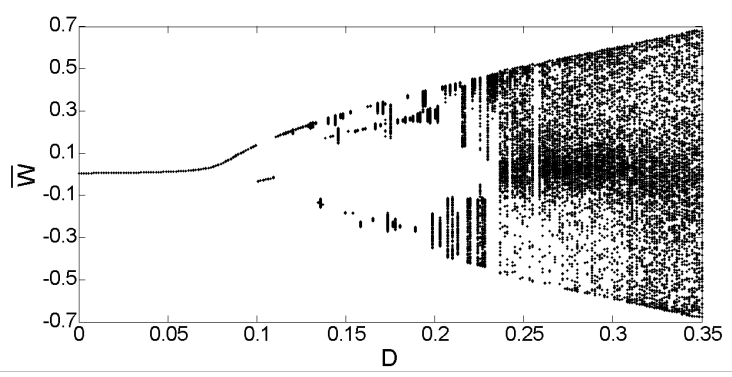

Fig. 3: Bifurcation diagram of the deflection with variation of the damage variable.

Base on the above analysis, damage effect plays a significant role in the bifurcation and chaotic behavior of the micro-plate system. The larger the damage variable is, the smaller the stiffness and the lower resistance ability of the structure will be, which result in instability and chaos of the micro-plate system. Therefore, with the increase of the damage value, the stability of the vibration for the microplate system will reduce.

\subsection{Influence of size effect}

This section aims at numerically investigating influence of size effect on nonlinear dynamics of the micro-plate system. Fig. 5 gives the bifurcation diagram of the micro-plate system when material length scale parameter is used as the control parameter with damage variable $D=0.13$ and damping coefficient $\bar{c}=0.003$.

Fig. 5 indicates that the system responses exhibit the alternation of periodic and chaotic motion. It can be seen from Fig. 5 that: 1) larger material length scale parame- 


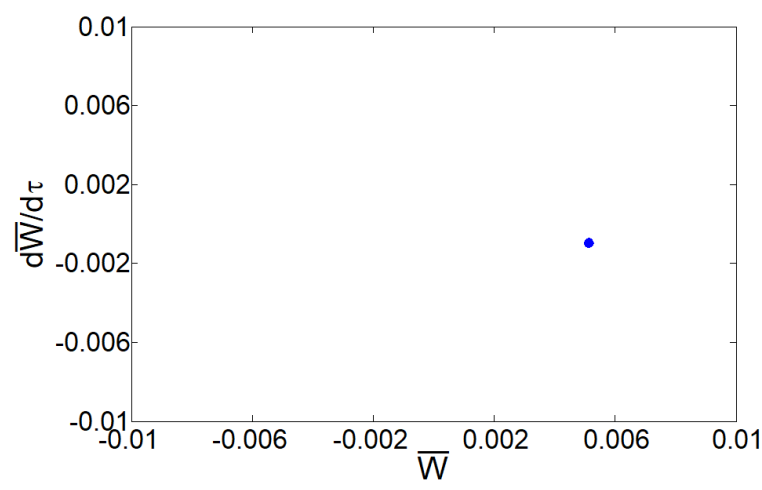

(a) Period-1 motion

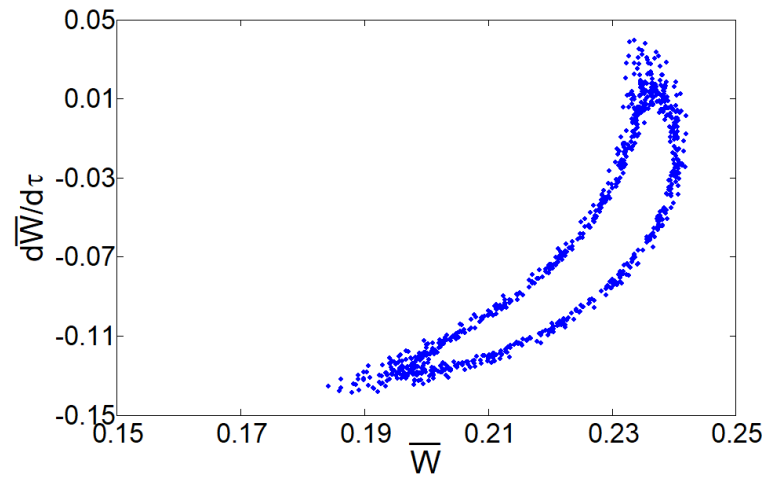

(b) Chaotic motion

Fig. 4: Poincare maps of the micro-plate system for different damage variable. (a) $D=0.003$ (b) $D=0.13$.

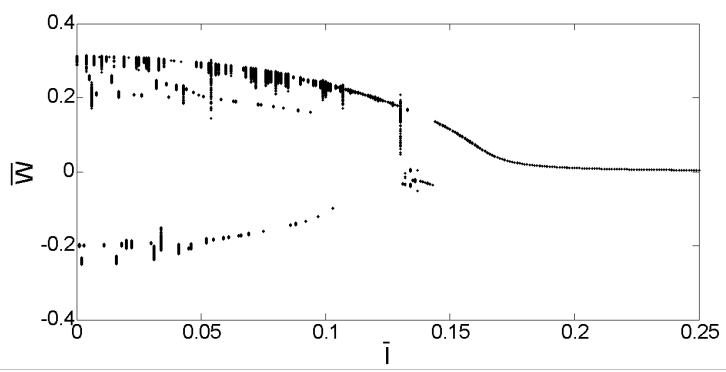

Fig. 5: Bifurcation diagram of the deflection with variation of material length scale parameter.

ter results in smaller middle point amplitude of vibration of the micro-plate; 2) the micro-plate system is becoming more and more stable as the larger material length scale parameter increases. As depicted in Fig. 6, the Poincare map shows fractal or chaotic characteristic which indicates chaotic motion of the system at $\bar{l}=0.01$ while it presents a single point which indicates periodic motion of the system at $\bar{l}=0.23$.

The above results indicate that the size effect induce the micro-plate additional rigidity which is important and cannot be neglected in the analysis of nonlinear behavior of micro-structure. Thus, size effect is conductive to the stability of the vibration for the micro-plate system.

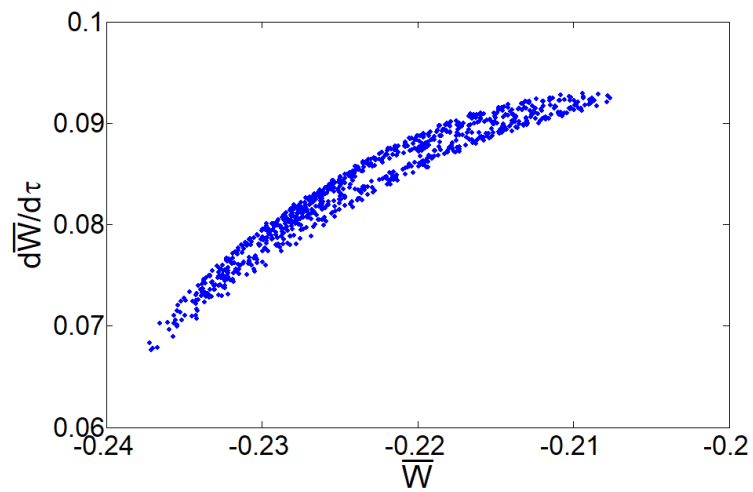

(a) Chaotic motion

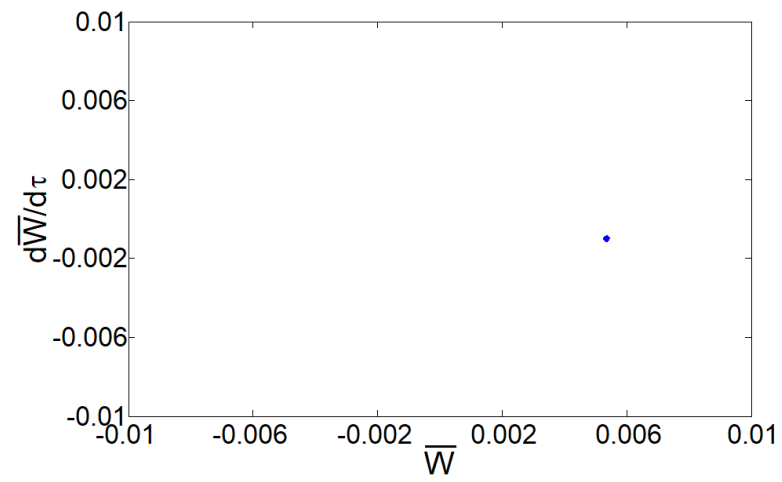

(b) Period-1 motion

Fig. 6: Poincare maps of the micro-plate system for different material length scale parameter. (a) $\bar{l}=0.01$ (b) $\bar{l}=0.23$.

\section{Conclusions}

In this work, a novel constitutive model of a sizedependent micro-plate with damage is developed on the basis of Talreja's tensor valued internal state damage variables and modified couple stress theory and further applied into analysis of nonlinear dynamics of a micro-plate system with electrostatic forces on both sides. The results show that the damage and size effect both have obvious influences on the bifurcation and chaos of the micro-plate system. With the increase of damage variable, the stability of the vibration for the micro-plate system will reduce. However, the increase of material length scale parameter will increase it. 
Acknowledgement: The authors would like to appreciate the anonymous reviewers for their enlightening comments and useful suggestions.

\section{References}

[1] C. Chen, H. Hu and L. Dai, "Nonlinear behavior and characterization of a piezoelectric laminated micro-beam system", Commun Nonlinear Sci Numer Simulat, 2013, 18(5), 1304-1315

[2] E. M. Miandodoab, H. N. Pishkenari, A. Yousefi-Koma and F. Tajaddodianfar, "Chaos prediction in NEMS-NEMS resonators", Int. J. Eng Sci, 2014, 82(3), 74-83

[3] M. I. Younis, "Multi-mode excitation of a clamped-clamped micro-beam resonator”, Nonlinear Dyn, 2015, 80(3), 1531-1541

[4] M. S. Siewe and U. H. Hegazy, "Homoclinic bifurcation and chaos control in MEMS resonators", Appl Math Model, 2011, 35(12), 5533-5552

[5] A. Seleim, S. Towfighian, E. Delande and E. Abdel-Rahman, "Dynamics of a close-loop controlled MEMS resonator", Nonlinear Dyn, 2012, 69(1-2), 615-633

[6] F. Tajaddodianfar, M. R. H. Yazdi, and H. N. Pishkenari, "Nonlinear dynamics of MEMS/NEMS resonators:analytical solution by the homotopy analysis method", Microsyst Technol, 2016 1-14

[7] W. Zhang and G. Meng, "Stability, bifurcation and chaos of a high-speed rub-impact rotor system in MEMS", Sensor Actuat A-Phys, 2006, 127(1), 163-178

[8] M. Gad-el-Hak, "The MEMS Handbook", CRC Press, Boca Raton, 2002

[9] X. P. Zhao, E. M. Abdel-Rahman and A. H. Nayfeh, “A reducedorder model for electrically actuated microplates", J. Micromecch Microeng, 2004, 14(7), 900-906

[10] S. Saghir and M. I. Younis, "An investigation of the static and dynamic behavior of electrically actuated rectangular microplates", Int. J. Nonlin Mech, 2016, 85, 81-93

[11] R. Batra, M. Porfiri and D. Spinello, "Effects of van der Waals force and thermal stresses on pull-in instability of clamped rectangular microplates”, Sensors, 2008, 8(2), 1048-1069

[12] R. Batra, M. Porfiri and D. Spinello, "Reduced-order models for microelectromechanical rectangular and circular plates incorporating the Casimir force”, Int. J. Solids Struct, 2008, 45(11-12), 3558-3583

[13] G. Chen and J. Chang, "Chaos in nonlinear dynamics of air bearing slider in contact”, Microsyst Technol, 2014, 20(8), 1739-1744

[14] E. M. Miandoab, H. N. Pishkenari and A. Yousei-Koma, "Nonlinear dynamics of nano-resonators: an analytical approach", Microsyst Technol., 2015 22(9), 1-13

[15] G. C. Tsiatas, "A new Kirchhoff plate model based on a modified couple stress theory”, Int. J. Solids Struct, 2009, 46(13), 2757-2764

[16] M. Tahani, A. R. Askari, Y. Mohandes and B. Hassani, “Sizedependent free vibration analysis of electrostatically predeformed rectangular micro-plates based on the modified couple stress theory”, Int. J. Mech Sci, 2015, 94-95(22), 185198
[17] F. Yang, A. C. M. Chong, D. C. C. Lam and P, Tong, "Couple stress based strain gradient theory for elasticity”, Int. J. Solids Struct, 2002, 39(10), 2731-2743

[18] A. R. Askari and M. Tahani, "Size-dependent dynamic pull-in analysis of geometric non-linear micro-plates based on the modified couple stress theory”, Physica E, 2017, 86, 262-274

[19] K. F. Wang, T. Kitamura and B. Wang, "Nonlinear pull-in instability and free vibration of micro/nanoscale plates with surface energy-a modified couple stress theory model”, Int. J. Mech Sci, 2015, 99(7), 288-296

[20] H. Farokhi and M. H. Ghayesh, "Nonlinear mechanics of electrically actuated microplates”, Int. J. Eng Sci, 2017, 123, 197213

[21] Y. F. Zheng, T. Chen and C. P. Chen, "A size-dependent model to study nonlinear static behavior of piezoelectric cantilever micro-beams with damage”, Microsyst Technol, 2016, 23(10), 1-8

[22] B. Jalalahmadi, F. Sadeghi and D.A. Peroulis, "Numerical Fatigue Damage Model for Life Scatter of MEMS Devices”, J. Micro-electromechs, 2009, 18(5), 1016-1031

[23] T. S. Slack, F. Sadeghi and P. A. Peroulis, "Phenomenological discrete brittle damage- mechanics model for fatigue of MEMS devices with application to LIGA Ni", J. Micro-electromechs, 2009,18(1), 119-128

[24] A. Basu, R. P. Hennessy, G. G. Adams and N. E. McGruer, "Hot switching damage mechanisms in MEMS contacts-evidence and understanding", J. Micromech Microeng, 2014, 24(10), 105004

[25] R. Talreja, "Damage Mechanics of Composite Materials", Elsevier, Amsterdam, 1994

[26] C. Chen, J. Yuan and Y. Mao, "Post-buckling of size-dependent micro-plate considering damage effects", J. Nonlinear Dyn, 2017, 90(2), 1301-1314

[27] P. Ladeveze and E. L. Dantec, "Damage modeling of the elementary ply for laminated composites", Compos Sci Technol, 1992, 43(5), 257-267

[28] Y. M. Fu, X. Q. Wang, "Analysis of Bifurcation and Chaos of the Piezoelectric Plate including Damage Effects", Int. J. Nonlin Sci Num, 2008, 9(1), 61-74

\section{A Appendix}

The expression for all the dimensionless coefficients of the governing equations shown in Eq. (34). $\mathrm{I}_{1}=\cos \pi \xi \sin ^{2} \pi \eta, \mathrm{I}_{2}=\sin ^{2} \pi \xi \cos \pi \eta, \mathrm{I}_{3}=\sin ^{2} \pi \xi \sin ^{2} \pi \eta$ $\Delta L_{11}=\iint_{2}\left[\Delta \bar{A}_{11} I_{1, \xi \xi}+\Delta \bar{A}_{66} \lambda_{1} I_{1, \eta \eta}-\beta \Delta \bar{G}_{1}\left(\lambda_{1}^{2} \lambda_{2} I_{1, \xi \xi \eta \eta}\right.\right.$ $\left.\left.+\lambda_{1}^{4} \lambda_{2} \mathrm{I}_{1, \eta \eta \eta \eta}\right)\right] \mathrm{I}_{1} d \xi d \eta$

$\Delta L_{12}=\iint_{s}\left[\Delta \bar{A}_{12} \lambda_{1} I_{2, \xi \eta}+\Delta \bar{A}_{66} \lambda_{1} I_{2, \xi \eta}+\beta \Delta \bar{G}_{1}\left(\lambda_{1} \lambda_{2} I_{2, \xi \xi \eta}\right.\right.$ $\left.\left.+\lambda_{1}^{3} \lambda_{2} \mathrm{I}_{2, \xi \eta \eta \eta}\right)\right] \mathrm{I}_{1} d \xi d \eta$

$\Delta L_{13}=\iint_{s}\left[\Delta \bar{A}_{11} I_{3, \xi} I_{3, \xi \xi}+\Delta \bar{A}_{12} \lambda_{1}^{2} I_{3, \eta} I_{3, \xi \eta}\right.$ $\left.+\Delta \bar{A}_{66} \lambda_{1}^{2}\left(I_{3, \xi} I_{3, \eta \eta}+\mathrm{I}_{3, \eta} \mathrm{I}_{3, \xi \eta}\right)\right] \mathrm{I}_{1} d \xi d \eta$

$\Delta L_{21}=\iint_{s}\left[\Delta \bar{A}_{12} \lambda_{1} I_{1, \xi \eta}+\lambda_{1} \Delta \bar{A}_{66} I_{1, \xi \eta}+\beta \Delta \bar{G}_{2}\left(\lambda_{1} \lambda_{2} I_{1, \xi \xi \xi \eta}\right.\right.$ $\left.\left.+\lambda_{1}^{3} \lambda_{2} \mathrm{I}_{1, \xi \eta \eta \eta}\right)\right] \mathrm{I}_{2} d \xi d \eta$ 


$$
\begin{aligned}
\Delta L_{22} & =\iint_{s}\left[\Delta \bar{A}_{22} \lambda_{1}^{2} I_{2, \eta}+\Delta \bar{A}_{66} I_{2, \xi \xi}-\beta \Delta \bar{G}_{2}\left(\lambda_{2} I_{2, \xi \xi \xi \xi}\right.\right. \\
\quad & \left.\left.+\lambda_{1}^{2} \lambda_{2} \mathrm{I}_{2, \xi \xi \eta \eta \eta}\right)\right] \mathrm{I}_{2} d \xi d \eta \\
\Delta L_{23} & =\iint_{s}\left[\Delta \bar{A}_{12} \lambda_{1} I_{3, \xi} I_{3, \xi \eta}+\Delta \bar{A}_{22} \lambda_{1}^{3} I_{3, \eta} I_{3, \eta \eta}\right. \\
& \left.+\Delta \bar{A}_{66} \lambda_{1}\left(I_{3, \eta} I_{3, \xi \xi}+\mathrm{I}_{3, \xi} \mathrm{I}_{3, \xi \eta}\right)\right] \mathrm{I}_{2} d \xi d \eta \\
\Delta L_{31} & =\iint_{s}\left[\left(\Delta \bar{D}_{11}+\beta \Delta \bar{G}_{3}\right) I_{3, \xi \xi \xi \xi}+\lambda_{1}^{2}\left(2 \Delta \bar{D}_{12} 2 \beta \Delta \bar{G}_{3}\right) \mathrm{I}_{3, \xi \xi \eta \eta}\right. \\
& \left.+4 \Delta \bar{D}_{66}+\lambda_{1}^{4}\left(\Delta \bar{D}_{22}+\beta \Delta \bar{G}_{3}\right) \mathrm{I}_{3, \eta \eta \eta \eta}\right] \mathrm{I}_{3} d \xi d \eta \\
\Delta L_{32} & =\iint_{s}\left[\Delta \bar{A}_{11} I_{1, \xi} I_{3, \xi \xi}+\Delta \bar{A}_{11} I_{1, \xi \xi} I_{3, \xi}+2 \Delta \bar{A}_{66} \lambda_{1}^{2} I_{1, \eta} I_{3, \xi \eta}\right. \\
& +\Delta \bar{A}_{66} \lambda_{1}^{2} \mathrm{I}_{1, \xi \eta} \mathrm{I}_{3, \eta}+\Delta \bar{A}_{12} \lambda_{1}^{2} \mathrm{I}_{1, \xi} \mathrm{I}_{3, \eta \eta} \\
& \left.+\Delta \bar{A}_{12} \lambda_{1}^{2} \mathrm{I}_{1, \xi \eta} \mathrm{I}_{3, \eta}+\lambda_{1}^{2} \Delta \bar{A}_{66} \mathrm{I}_{1, \eta \eta} \mathrm{I}_{3, \xi}\right] \mathrm{I}_{3} d \xi d \eta
\end{aligned}
$$

$$
\begin{aligned}
\Delta L_{33}= & \iint_{s}\left[\Delta \bar{A}_{12} \lambda_{1} I_{2, \eta} I_{3, \xi \xi}+\Delta \bar{A}_{12} \lambda_{1} I_{2, \xi \eta} I_{3, \xi}+\right. \\
& 2 \lambda_{1} \Delta \bar{A}_{66} \mathrm{I}_{2, \xi} \mathrm{I}_{3, \xi \eta}+\Delta \bar{A}_{66} \lambda_{1} \mathrm{I}_{2, \xi \xi} \mathrm{I}_{3, \eta}+\lambda_{1}^{3} \Delta \bar{A}_{22} \mathrm{I}_{2, \eta} \mathrm{I}_{3, \eta \eta} \\
& \left.+\lambda_{1}^{3} \Delta \bar{A}_{22} \mathrm{I}_{2, \eta \eta} \mathrm{I}_{3, \eta}+\Delta \bar{A}_{66} \lambda_{1} \mathrm{I}_{2, \xi \eta} \mathrm{I}_{3, \xi}\right] \mathrm{I}_{3} d \xi d \eta \\
\Delta L_{34}= & \iint_{s}\left[\frac{\Delta \bar{A}_{11}}{2} I_{3, \xi} I_{3, \xi} I_{3, \xi \xi}+\frac{\Delta \bar{A}_{12} \lambda_{1}^{2} I_{3, \eta} I_{3, \eta} I_{3, \xi \xi}}{2}\right. \\
& +\Delta \bar{A}_{11} \mathrm{I}_{3, \xi} \mathrm{I}_{3, \xi} \mathrm{I}_{3, \xi \xi}+\Delta \bar{A}_{12} \lambda_{1}^{2} \mathrm{I}_{3, \xi} \mathrm{I}_{3, \eta} \mathrm{I}_{3, \xi \eta} \\
& +2 \Delta \bar{A}_{66} \lambda_{1}^{2} \mathrm{I}_{3, \xi} \mathrm{I}_{3, \eta} \mathrm{I}_{3, \xi \eta}+\Delta \bar{A}_{66} \lambda_{1}^{2} \mathrm{I}_{3, \eta} \mathrm{I}_{3, \eta} \mathrm{I}_{3, \xi \xi} \\
& +\Delta \bar{A}_{66} \lambda_{1}^{2} \mathrm{I}_{3, \xi} \mathrm{I}_{3, \eta} \mathrm{I}_{3, \xi \eta}+\frac{\Delta \bar{A}_{12}}{2} \lambda_{1}^{2} \mathrm{I}_{3, \xi} \mathrm{I}_{3, \xi} \mathrm{I}_{3, \eta \eta} \\
& +\frac{\Delta \bar{A}_{62}}{2} \lambda_{1}^{4} \mathrm{I}_{3, \eta} \mathrm{I}_{3, \eta} \mathrm{I}_{3, \eta \eta}+\Delta \bar{A}_{12} \lambda_{1}^{2} \mathrm{I}_{3, \xi} \mathrm{I}_{3, \eta} \mathrm{I}_{3, \xi \eta} \\
& +\Delta \bar{A}_{22} \lambda_{1}^{4} I_{3, \eta} \mathrm{I}_{3, \eta} \mathrm{I}_{3, \eta \eta}+\Delta \bar{A}_{66} \lambda_{1}^{2} \mathrm{I}_{3, \xi} \mathrm{I}_{3, \eta} \mathrm{I}_{3, \xi \eta} \\
& \left.+\Delta \bar{A}_{66} \lambda_{1}^{2} \mathrm{I}_{3, \xi} \mathrm{I}_{3, \xi} \mathrm{I}_{3, \eta \eta}\right] \mathrm{I}_{3} d \xi d \eta
\end{aligned}
$$

